\title{
Surfaces of Low Degree Containing a Canonical Curve
}

\author{
Izzet Coskun
}

\begin{abstract}
In this paper, we generalize a classical theorem of del Pezzo [D] and Fujita $[\mathbf{F 1}]$ and a recent theorem of Casnati $[\mathbf{C a}]$ about low degree surfaces containing a canonical curve $C_{c a n}$. Every canonical curve of genus $g$ is contained in a surface of degree less than or equal to $2 g-3$. We study canonical curves that are contained in a surface of degree smaller than $2 g-3$. Fix an integer $\kappa \geq-1$. Our main theorem is that if $g>3 \kappa+12$ and $(g, \kappa) \neq(10,-1)$, then the minimal degree surface $S$ containing $C_{\text {can }}$ has degree $d=g+\kappa$ if and only if $\kappa$ is odd and $C$ is a double cover $f: C \rightarrow B$ of a curve $B$ of genus $\frac{\kappa+3}{2}$. In this case, $S$ is the image of $X=\mathbb{P}\left(f_{*} K_{C}\right)$ under the linear system $\left|\mathcal{O}_{X}(1)\right|$. Our methods also apply to curves embedded with complete linear systems of high degree.
\end{abstract}

\section{Introduction}

Let $C$ be a smooth, projective, non-hyperelliptic curve of genus $g$ over the complex numbers $\mathbb{C}$. The canonical linear system $\left|K_{C}\right|$ embeds $C$ in $\mathbb{P}^{g-1}$ as a non-degenerate curve $C_{c a n}$ of degree $2 g-2$. The extrinsic geometry of $C_{c a n}$ closely reflects the intrinsic geometry of $C$. In this paper, we explore the implications of the existence of a low degree surface containing $C_{c a n}$ for the intrinsic geometry of $C$.

Let $p$ be a point on $C_{c a n}$. Let $S$ be the surface obtained by taking the cone over $C_{c a n}$ with vertex at $p$. Then $S$ has degree $2 g-3$. Therefore, every canonical curve is contained in a surface of degree less than or equal to $2 g-3$. Ciliberto and Harris $[\mathbf{C H}]$ prove that if $g \geq 23$ and $C$ is general in moduli, then a minimal degree surface $X$ containing $C_{\text {can }}$ has degree $2 g-3$ and $X$ is a cone over $C_{c a n}$ with vertex on $C_{c a n}$. The purpose of this paper is to study the geometry of canonical curves that are contained in surfaces of degree less than $2 g-3$.

Let $X$ be an irreducible, non-degenerate variety of degree $d$ and dimension $r$ in $\mathbb{P}^{n}$. Then the invariants $d, r$ and $n$ satisfy the inequality $d+r-1 \geq n$.

2000 Mathematics Subject Classification. Primary 14N25; Secondary 14H51, 14H30, 14N05.

Key words and phrases. Canonical curve, low degree surface.

During the preparation of this article the author was partially supported by the NSF grant DMS-0737581, the NSF-CAREER grant DMS-0950951535 and an Alfred P. Sloan Foundation Fellowship. 
Varieties for which $d+r-1-n$ is small have been classified by Bertini, Castelnuovo, del Pezzo, Fujita, Ionescu, Livorni among many others (see $[\mathbf{B e}],[\mathbf{D}],[\mathbf{E H}],[\mathbf{F 1}],[\mathbf{F 2}],[\mathbf{F 3}],[\mathbf{I o}],[\mathbf{L 1}],[\mathbf{L 2}])$. For example, a classical theorem of Bertini and del Pezzo $[\mathbf{E H}]$ asserts that varieties that satisfy $d+r-1=n$ are quadric hypersurfaces, the Veronese surface in $\mathbb{P}^{5}$, rational normal scrolls and cones over these varieties.

Since $C_{c a n}$ is non-degenerate, any surface containing $C_{c a n}$ is also nondegenerate and has degree at least $g-2$. If $C_{c a n}$ is contained in a surface $S$ of degree $g-2$, then, by the classification of varieties of minimal degree $[\mathbf{E H}], S$ is either a rational normal scroll or the Veronese surface in $\mathbb{P}^{5}$. Moreover, by the Babbage-Enriques-Petri Theorem ([ACGH, p.124, 131]), $C$ is either trigonal or isomorphic to a plane quintic curve. In particular, if $g>6$, then $C$ is trigonal.

If the minimal degree surface $S$ containing $C_{c a n}$ has degree $g-1$, then $S$ is either a del Pezzo surface (i.e., the anti-canonical image of a quadric surface in $\mathbb{P}^{3}$ or a blow-up of $\mathbb{P}^{2}$ in less than or equal to 5 possibly infinitely near points) or a cone over an elliptic normal curve of degree $g-1$ in $\mathbb{P}^{g-2}[\mathbf{F 1}]$. If $S$ is a del Pezzo surface, then $C$ is birational to a complete intersection $(2,4)$ in $\mathbb{P}^{3}$ or to a plane sextic curve with $10-g$ possibly infinitely near double points. Otherwise, $C$ is bi-elliptic, that is, $C$ admits a two-to-one map to an elliptic curve. In particular, if $g>10$, then $C$ is bi-elliptic.

Casnati $[\mathbf{C a}]$ proves that if the minimal degree surface $S$ containing $C_{c a n}$ has degree $g$, then $7 \leq g \leq 12$. In these cases, $S$ is a conic bundle over $\mathbb{P}^{1}$ whose fibers cut out the unique $g_{4}^{1}$ on $C$. In particular, if $g>12$, then $C_{c a n}$ is not contained in a surface of degree $g$.

These examples suggest that if the genus is large enough, the existence of a surface of low degree containing $C_{c a n}$ implies that $C$ is a small degree cover of a curve of low genus. Our first theorem makes this precise.

Theorem 1.1. Fix $\kappa \geq-1$. Assume that the minimal degree surface $S$ containing a canonical curve $C_{\text {can }}$ of genus $g$ has degree $d=g+\kappa<2 g-3$ and that $(g, \kappa) \neq(10,-1)$.

(1) If $\kappa$ is even, then $g \leq 3 \kappa+12$.

(2) If $\kappa$ is odd, then either $g \leq 3 \kappa+12$ or $C$ admits a two-to-one map $f: C \rightarrow B$ to a curve $B$ of genus $\frac{\kappa+3}{2}$. If $g>3 \kappa+12$, then $S$ is the image of the ruled surface $X=\mathbb{P}\left(f_{*} K_{C}\right)$ over $B$ under the linear system $\left|\mathcal{O}_{X}(1)\right|$.

(3) Conversely, if $C$ is a double cover of a curve of genus $\frac{\kappa+3}{2}$, then $C_{c a n}$ is contained in a surface of degree $g+\kappa$.

Remark 1.2. When $\kappa=-1$, we recover the theorem of del Pezzo [D] and Fujita $[\mathbf{F 1}]$ that a curve of $g>10$ which lies on a surface of degree $g-1$ is bi-elliptic.

When $\kappa=0$, we recover Casnati's bound that if the minimal degree surface containing $C_{c a n}$ has degree $g$, then $7 \leq g \leq 12$. Here the lower 
bound is trivial since curves of degree $g \leq 6$ always lie on a surface of degree $g-1$ or less (see Example 1.7).

REMARK 1.3. After I wrote this paper, I became aware that Casnati in $[\mathbf{C a 2}]$, independently and using different techniques, proved the case $\kappa=1$ of Theorem 1.1.

REMARK 1.4. Let $C$ be a smooth, sextic plane curve. Then the genus of $C$ is 10 and $C_{c a n}$ is contained in the three-uple Veronese embedding of $\mathbb{P}^{2}$. The curve $C$ cannot be bi-elliptic since its gonality is five instead of four. Therefore, we conclude that there are canonical curves of genus 10 that are not bi-elliptic and lie on a surface of degree 9 . This example explains the need to exclude the case $(g, \kappa)=(10,-1)$ in Theorem 1.1.

REMARK 1.5. Let $C$ be a curve of type $(4, r+2)$, with $r \geq 2$, on $\mathbb{P}^{1} \times \mathbb{P}^{1}$. Then $C$ has genus $3 r+3$. A general such curve has trivial automorphism group, hence it cannot be a double cover of a curve of lower genus. Consider the embedding $\phi: \mathbb{P}^{1} \times \mathbb{P}^{1} \rightarrow \mathbb{P}^{3 r+2}$ given by the linear system $\left|\mathcal{O}_{\mathbb{P}^{1} \times \mathbb{P}^{1}}(2, r)\right|$. Then the image of $\phi$ is a surface of degree $4 r$. Furthermore, $\phi$ embeds $C$ as the canonical curve $C_{\text {can }}$. In this case, we have that $\kappa=4 r-(3 r+3)=r-3$. Hence $g=3 r+3=3 \kappa+12$. If $C$ is general, then $C_{c a n}$ cannot be contained in a surface of degree less than $4 r$. Otherwise, by Theorem 1.1, $C$ would be a double cover of a curve of genus less than $r / 2$. We conclude that the bounds in Theorem 1.1 are sharp.

We will say that a curve $C \subset \mathbb{P}^{n}$ is cut out by quadrics, if the homogeneous ideal of $C$ is generated by quadratic equations. Our proof of Theorem 1.1 applies more generally to curves $C$ that are embedded by non-special complete linear systems and are cut out by quadrics. For example, these assumptions are satisfied when $C$ is embedded by a complete linear system of degree $s \geq 2 g+2$ [ACGH, p. 143]. For simplicity, we will restrict ourselves to this case. By taking a cone over $C$ with a vertex on $C$, it is clear that every curve of degree $s$ is contained in a surface of degree $s-1$. Our next theorem studies the curves that are contained in a surface of strictly smaller degree.

TheOrem 1.6. Fix $\kappa \geq-1$. Let $C$ be a curve of genus $g$ and degree $s>2 g+1$ embedded in $\mathbb{P}^{s-g}$ by a complete linear system. Assume that the minimal degree surface containing $C$ has degree $d=s-g+\kappa<s-1$ and that $(\kappa, s-g) \neq(0,9)$.

(1) If $\kappa$ is even, then $s-g \leq 3 \kappa+9$.

(2) If $\kappa$ is odd, then either $s-g \leq 3 \kappa+9$ or $C$ admits a two-to-one map to a curve of genus $\frac{\kappa+1}{2}$.

It is interesting to classify curves $C$ of genus $g \leq 3 \kappa+12$ whose canonical models $C_{c a n}$ are contained in a surface of degree $g+\kappa$. The following examples demonstrate that as the genus increases this classification becomes more intricate. 
EXAMPLE 1.7. - Every canonical curve of genus 4 is contained in a quadric surface. The quadric surface is smooth if $C$ admits two distinct $g_{3}^{1}$ 's. Otherwise, the surface is a quadric cone $[\mathbf{A C G H}]$.

- A canonical curve of genus 5 is contained in a three-dimensional linear system of quadric hypersurfaces. If $C$ is trigonal, then these quadrics cut out a cubic scroll and $C_{c a n}$ is contained in a surface of degree 3. Otherwise, the minimal degree surface containing $C_{c a n}$ has degree 4 and can be obtained as a complete intersection of two of the quadric hypersurfaces containing $C_{\text {can }}[\mathbf{A C G H}]$.

- A general curve $C$ of genus 6 can be realized in $\mathbb{P}^{2}$ as a curve of degree 6 with four nodes. The blow-up of $\mathbb{P}^{2}$ at the nodes embeds in $\mathbb{P}^{5}$ as a del Pezzo surface of degree 5 by the anti-canonical map and contains $C_{\text {can }}[\mathbf{A C G H}]$. Therefore, every canonical curve of genus 6 is contained in a surface of degree 4 or 5 . The curve is contained in a surface of degree 4 if and only if it is trigonal or isomorphic to a smooth plane quintic curve [ACGH]. Otherwise, the minimal degree surface containing $C_{c a n}$ has degree 5 .

- A general curve $C$ of genus 7 can be realized in $\mathbb{P}^{2}$ as a curve of degree 7 with eight nodes. The blow-up of $\mathbb{P}^{2}$ at the nodes of $C$ embeds into $\mathbb{P}^{6}$ by the linear system of quartic curves vanishing at the nodes of $C$. The surface has degree 8 and contains $C_{c a n}$ [ACGH]. We, therefore, conclude that the minimal degree surface containing a canonical curve of genus 7 can have degree $5,6,7$ or 8. The minimal degree surface containing $C_{c a n}$ has degree 5 if and only if $C$ is trigonal. The minimal degree surface containing $C_{c a n}$ has degree 6 if and only if $C$ can be realized as a plane sextic or $C$ is bi-elliptic $[\mathbf{A C G H}]$. If the minimal degree surface containing $C_{c a n}$ has degree 7 , then $S$ is a conic bundle over $\mathbb{P}^{1}$ and $C$ has a unique $g_{4}^{1}[\mathbf{C a}]$. For the general canonical curve of genus 7 , the minimal degree surface containing $C_{c a n}$ has degree 8.

In the last section, using the classification of surfaces of low degree, we will make some remarks about curves $C$ such that $C_{c a n}$ is contained in a surface of degree $g+\kappa$ for small values of $\kappa$.

The organization of this paper is as follows. In the next section, we will recall some basic facts about ruled surfaces and the $\Delta$-genus that are used in the proof. In $\S 3$, we will prove Theorems 1.1 and 1.6. In the final section, we will study the geometry of surfaces of degree $g+\kappa$ containing a canonical curve for small values of $\kappa$.

Acknowledgements: I would like to thank Gianfranco Casnati, Lawrence Ein and Mihnea Popa for invaluable comments. This paper was inspired by the papers of Ciliberto and Harris $[\mathbf{C H}]$ and Casnati $[\mathbf{C a}]$. I would also like to thank the organizers of the AMS Special Session on Computational Algebraic and Analytic Geometry, Mika Seppälä, Tanush Shaska and Emil Volcheck, for giving me a chance to present my work at the Joint Meetings. 


\section{The background on ruled surfaces and $\Delta$-genus}

In this section, we recall some basic facts concerning ruled surfaces and the $\Delta$-genus. We refer the reader to $[\mathbf{B}, \S \mathrm{III}]$ and $[\mathbf{H}, \mathrm{V} .2]$ for more details on ruled surfaces and to $[\mathbf{F 3}],[\mathbf{F} 4]$ and $[\mathbf{H o}]$ for more details on the $\Delta$-genus.

Let $X$ be a smooth projective variety of dimension $r$. Let $L$ be a basepoint-free line bundle on $X$ of degree $d$ whose complete linear system gives rise to a birational morphism $\rho_{L}: X \rightarrow \mathbb{P}^{n}$. Recall that the $\Delta$-genus, first introduced by Fujita (see $[\mathbf{F 4}]$ ), is defined by

$$
\Delta=\Delta(X, L)=d+r-h^{0}(X, L) .
$$

To prove Theorems 1.1 and 1.6, we will use a rough classification of surfaces by $\Delta$-genus due to Tony Horowitz.

Recall that a surface $S$ is birationally ruled if it is birational to $B \times \mathbb{P}^{1}$ for some curve $B$. A surface $S$ is geometrically ruled if $S$ admits a morphism $\pi: S \rightarrow B$ to a curve such that the fibers are all isomorphic to $\mathbb{P}^{1}$. A geometrically ruled surface is isomorphic to the projectivization of a rank 2 vector bundle $E$ over $B[\mathbf{H}, \mathrm{V} .2 .2]$. A surface $S$ is projectively ruled if $S$ is the birational image in projective space of a geometrically ruled surface such that the fibers are mapped to lines.

We will reduce the proofs of Theorems 1.1 and 1.6 to the following Theorem of Horowitz.

Theorem $\mathbf{A}$ of $[\mathbf{H o}]$. Let $X$ be a surface and let $L$ be a line bundle on $X$ as above. If $(X, L) \neq\left(\mathbb{P}^{2}, \mathcal{O}_{\mathbb{P}^{2}}(3)\right)$ and $3 \Delta(X, L)+6<h^{0}(X, L)$, then $\rho_{L}(X)$ is projectively ruled.

If $S$ is a geometrically ruled surface $\mathbb{P} E$ over a curve $B, E$ can be normalized so that $H^{0}(B, E) \neq 0$, but for any line bundle of negative degree $L$ on $B, H^{0}(B, E \otimes L)=0$. When $E$ is so normalized, the degree $e$ of $E$ is an invariant of $S$ and there exists a section $C_{0}$ of the projective bundle with $C_{0}^{2}=e[\mathbf{H}, \mathrm{V} .2 .8]$. From now on, we will always assume that $E$ has been so normalized and $C_{0}$ is a section with $C_{0}^{2}=e$. We caution the reader that Hartshorne uses $-e$ instead of $e$.

Lemma 2.1. Let $\pi: S=\mathbb{P} E \rightarrow B$ be a geometrically ruled surface over a curve $B$ of genus $h$. Let $L$ be a line bundle of degree d on $S$. Let $C$ be a curve of genus $g$ on $S$ that defines a bi-section of $\pi$. Let $V \subset H^{0}(S, L)$ be a base-point-free linear system that defines a birational map $\phi_{V}$ of $S$ onto a projectively ruled surface in $\mathbb{P}^{n}$.

(1) If $n=g-1$ and $\phi_{V}$ restricted to $C$ is the canonical embedding of $C$, then

$$
h=\frac{d-g+3}{2} .
$$


(2) If $n=s-g$ and $V$ restricted to $C$ is a non-special, complete linear system on $C$ of degree $s$, then

$$
h=\frac{d+g-s+1}{2} .
$$

Proof. Let $C_{0}$ be the section on $S$ with $C_{0}^{2}=e$. The Neron-Severi space of $S$ is generated by the numerical equivalence classes of $C_{0}$ and a fiber $F$ of $\pi$. By adjunction, the canonical class of $S$ is numerically equivalent to

$$
K_{S} \equiv-2 C_{0}+(2 h+e-2) F .
$$

By assumption, the image of $\phi_{V}$ is a geometrically ruled surface of degree $d$. Therefore, the degree of $L$ on $F$ is one. If we express the numerical equivalence class of $L \equiv C_{0}+m F$, writing $L^{2}=e+2 m=d$, we see that $L$ is numerically equivalent to

$$
L \equiv C_{0}+\frac{d-e}{2} F
$$

On the other hand, since $C$ is a bi-section of $\pi, C$ is numerically equivalent to $C \equiv 2 C_{0}+r F$. We can compute $r$ in two different ways. First, the degree of the curve is $s$. Hence,

$$
s=L \cdot C=2 e+(d-e)+r .
$$

We conclude that the numerical equivalence class of $C$ is

$$
C \equiv 2 C_{0}+(s-d-e) F .
$$

In particular, when $\phi_{V}$ restricts to the canonical linear system on $C, C \equiv$ $2 C_{0}+(2 g-2-d-e) F$. On the other hand, by the adjunction formula,

$$
\operatorname{deg}\left(K_{C}\right)=\left(K_{S}+C\right) \cdot C .
$$

Hence,

$$
2 g-2=2(2 h-2+s-d) .
$$

We conclude that

$$
h=\frac{d+g-s+1}{2} .
$$

In particular, when $\phi_{V}$ restricts to the canonical linear system on $C$, then

$$
h=\frac{d-g+3}{2} \text {. }
$$

Let $f: C \rightarrow B$ be a two-to-one morphism from a smooth, projective curve $C$ of genus $g$ to a smooth, projective curve $B$ of genus $h$. Given a line bundle $L$ of degree $d$ on $C, f_{*} L$ is a vector bundle of rank two on $B$. By the Riemann-Roch Theorem, the vector bundle $f_{*} L$ has degree $d+2 h-g-1$. The surface $\mathbb{P}\left(f_{*} L\right)$ is a geometrically ruled surface over $B$.

In this paper, we will be especially interested in the case when $L$ is the canonical line bundle $K_{C}$. The vector bundle $f_{*} K_{C}$ is a rank two bundle on 
$B$ of degree $2 h+g-3$. In this case, by duality, $\left(f_{*} K_{C}\right)^{*} \otimes K_{B}=f_{*} \mathcal{O}_{C}$. The natural inclusion of $\mathcal{O}_{B}$ in $f_{*} \mathcal{O}_{C}$ leads to an exact sequence

$$
0 \rightarrow \mathcal{O}_{B} \rightarrow f_{*} \mathcal{O}_{C} \rightarrow \mathcal{O}_{B}(-D) \rightarrow 0,
$$

where $D$ is half of the ramification divisor of $f$. For simplicity, set $M=$ $\mathcal{O}_{B}(D)$. The norm map splits this sequence leading to the equality $f_{*} \mathcal{O}_{C}=$ $\mathcal{O}_{B} \oplus M^{-1}$. By duality, we conclude that $f_{*} K_{C}=K_{B} \oplus\left(K_{B} \otimes M\right)$.

Therefore, the ruled surface $X=\mathbb{P}\left(f_{*} K_{C}\right)$ over $B$ has invariant $e=$ $2 h-g-1$. The curve $C$ naturally embeds in $X$. The linear system $\left|\mathcal{O}_{X}(1)\right|$ gives rise to the map $\phi: X \rightarrow \mathbb{P}^{g-1}$. The image of $\phi$ is a surface of degree $2 h+g-3$. The map $\phi$ restricts to the canonical map on $C$.

The inclusion $K_{B} \hookrightarrow f_{*} K_{C}$ gives rise to a section $B_{0}$ of $X$ over $B$. The effective cone of $X$ is generated by the class of $B_{0}$ and the class of a fiber $[\mathbf{H}, \mathrm{V} .2 .20]$. Therefore, when $h>1, \mathcal{O}_{X}(1)$ is ample since it has positive degree on both generators of the effective cone. The map $\phi$ restricts to the canonical map on $B_{0}$. Hence, $\mathcal{O}_{X}(1)$ is not very ample if $B$ is hyperelliptic. In fact, if $B$ is hyperelliptic, the image of $X$ under $\phi$ is not normal since $\phi$ maps $B_{0}$ two-to-one onto a rational normal curve of degree $2 h-2$. On the other hand, if $g>2 h+1$ and $B$ is not hyperelliptic, then $\mathcal{O}_{X}(1)$ is very ample and $\phi$ is an embedding. There are sections $B_{1}$ of $X$ that are disjoint from $B_{0}$ induced by the inclusion $K_{B} \otimes M \hookrightarrow f_{*} K_{C}$. If $g>2 h+1, \phi$ embeds both $B_{0}$ and $B_{1}$ into $\mathbb{P}^{g-1}$ as curves with disjoint spans. The scroll over these two curves is smooth. Hence, it follows that $\phi$ is an embedding.

\section{Minimal degree surfaces containing a canonical curve}

In this section, we prove Theorem 1.1 and Theorem 1.6.

Proof of Theorem 1.1. Let $\kappa \geq-1$. In Example 1.7, we saw that the canonical image of every non-hyperelliptic curve of genus $g \leq 6$ is contained in a surface of degree $g-1$ or $g-2$. We can, therefore, assume that $g>6$. Let $S$ be a surface in $\mathbb{P}^{g-1}$ of degree $d=g+\kappa$ containing $C_{c a n}$. Then, by the Babbage-Enriques-Petri Theorem, $C_{c a n}$ is cut out by quadrics unless $C$ is trigonal [ACGH]. If $C$ is trigonal, then $C_{c a n}$ lies on a rational normal surface scroll of degree $g-2$. Since by assumption the degree of the minimal surface containing $C_{c a n}$ has degree $g+\kappa>g-2$, we conclude that $C_{c a n}$ is cut out by quadrics.

Take a quadric $Q$ containing $C_{c a n}$ but not $S$. By Bezout's Theorem, $S \cap Q$ has degree $2 d$. Since $d<2 g-3, S$ cannot be everywhere singular along $C_{c a n}$. Let $X$ be the minimal desingularization of $S$. Let $L$ be the pull-back of $\mathcal{O}_{S}(1)$ to $X$. Denote the proper transform of $C$ in $X$ again by $C$.

We first bound the $\Delta$-genus of the pair $(X, L)$.

$$
\Delta(X, L)=d+2-h^{0}(X, L) \leq d-g+2=\kappa+2 .
$$


If $g>3 \kappa+12$, then

$$
3 \Delta+6=3(\kappa+2)+6=3 \kappa+12<g \leq h^{0}(X, L) .
$$

By Horowitz's Theorem [Ho, Theorem A], if $g>3 \kappa+12$ and $(X, L) \neq$ $\left(\mathbb{P}^{2}, \mathcal{O}_{\mathbb{P}^{2}}(3)\right)$, then $S$ is projectively ruled. Hence $\pi: X \rightarrow B$ is a geometrically ruled surface. From now on we assume that $g>3 \kappa+12$ and $(g, \kappa) \neq(10,-1)$. We may, therefore, assume that $S$ is projectively ruled.

Since $C_{c a n}$ is cut out by quadrics, $C_{c a n}$ is contained in the intersection of $S$ by a quadric. Consequently, $C$ is either a bi-section or a section of $\pi: X \rightarrow B$. If $C$ is a bi-section, then by Lemma 2.1 , the genus $h$ of the curve $B$ is related to $g$ and $d$ by the formula

$$
h=\frac{d-g+3}{2}=\frac{\kappa+3}{2} .
$$

Since the genus is an integer, $\kappa$ must be odd.

If $C$ is in a section class, then $B$ is isomorphic to $C$. Take a general irreducible hyperplane section $H$ of $S$. The curve $H$ has degree $d$ and spans a projective linear space of dimension $g-2$ and the normalization of $H$ is isomorphic to $C$. Let $D$ be the hyperplane divisor on the normalization of $H$. Clifford's Theorem says that $h^{0}(D)-1 \leq \operatorname{deg}(D) / 2$ for any special divisor on a curve $Y$ of genus $g$ with equality when $D=\mathcal{O}_{Y}$ or $D=K_{Y}$ or when $Y$ is a hyperelliptic curve and $D$ is $m g_{2}^{1}$ for $0<m<g$ [ACGH]. Applying Clifford's Theorem to the normalization of $H$, we obtain that if $d<2 g-4$, then $g-2>d / 2$. Hence, the hyperplane divisor is non-special. However, by the Riemann-Roch Theorem, $h^{0}(D)=d-g+1<g-3$. Hence, the image $H$ cannot span $\mathbb{P}^{g-2}$. We thus obtain a contradiction. On the other hand, if $d=2 g-4$, then, using the fact that $d=g+\kappa$, we obtain that $\kappa=g-4$. The inequality $g>3 \kappa+12$, leads to the inequality $-4>\kappa$, which is a contradiction. We conclude that $C$ cannot be in a section class on $X$. Therefore, it must be in a bi-section class.

By Lemma 2.1, we conclude that $C$ admits a two-to-one map to $B$, a curve of genus $(\kappa+3) / 2$. As observed above, since the genus is an integer, $\kappa$ must be odd. This concludes the proof of Part (1) and the first statement in Part (2) of Theorem 1.1.

Conversely, suppose $C$ admits a two-to-one map $f: C \rightarrow B$ to a curve of genus $h=(\kappa+3) / 2$. Then, as observed in $\S 2, E=f_{*} K_{C}$ is a rank two vector bundle on $B$ of degree $g+2 h-3$. The curve $C$ embeds into the geometrically ruled surface $\mathbb{P} E$ over $B$. The line bundle $\mathcal{O}_{\mathbb{P} E}(1)$ gives a map from $\mathbb{P} E \rightarrow \mathbb{P}^{g-1}$ onto a projectively ruled surface of degree $g+2 h-3=g+\kappa$. Furthermore, the restriction of $\mathcal{O}_{\mathbb{P} E}(1)$ to $C$ is the canonical linear series on $C$. Therefore, if $C$ is a double cover of a curve of genus $\frac{\kappa+3}{2}$, then $C_{c a n}$ is contained in a surface of degree $g+\kappa$. As an aside, observe that whether $B$ is hyperelliptic is determined from the singularities of the surface.

The map $f: C \rightarrow B$ determines the surface $S$. The curve $C$ is a bi-section of the projectively ruled surface. If $q_{1}, q_{2}$ are the two points in 
$f^{-1}(p)$ for $p \in B$, then $S$ contains the line $\overline{q_{1} q_{2}}$ spanned by $q_{1}$ and $q_{2}$. As the point $p$ varies over $B$, the lines spanned by the points in $f^{-1}(p)$ sweep out the surface $S$. Hence, $S$ can be recovered from the map $f$. The image of $X=\mathbb{P}\left(f_{*} K_{C}\right)$ in $\mathbb{P}^{g-1}$ under the map $\phi$ defined by the linear system $\left|\mathcal{O}_{X}(1)\right|$ is a projectively ruled surface swept out by the lines spanned by the pairs of points on $C_{c a n}$ that are mapped to the same point on $B$ by $f$. Hence, $S$ is the image of $X$ under $\phi$. This concludes the proof of Theorem 1.1.

Proof of Theorem 1.6. The proof of Theorem 1.6 requires only minor modification. Let $S$ be a surface of degree $d=s-g+\kappa$ in $\mathbb{P}^{s-g}$ containing $C$. Let $X$ be the minimal desingularization of $S$ and let $L$ be the pull-back of $\mathcal{O}_{\mathbb{P}^{s-g}}(1)$ on $X$. The $\Delta$-genus of $(X, L)$ can be calculated as follows.

$$
\Delta=\Delta(X, L) \leq s-g+\kappa+2-(s-g+1)=\kappa+1 .
$$

Hence, if $s-g+1>3 \kappa+9$, then $3 \Delta+6 \leq 3 \kappa+9<s-g+1 \leq h^{0}(X, L)$. Therefore, by Horowitz's Theorem [Ho, Theorem A], $S$ is projectively ruled.

Since, by assumption $C$ is cut out by quadrics, then $C$ is either a section or a bi-section of $X \rightarrow B$. As in the proof of Theorem 1.1, Clifford's Theorem implies that $C$ cannot be a section. If $C$ were a section, then $B$ and $C$ would be isomorphic. Let $H$ be a general hyperplane section of $S$. The normalization $H^{\nu}$ of $H$ would also be isomorphic to $C$. By assumption, $\kappa<g-1$. The inequality $s-g-1 \leq \frac{1}{2}(s-g+\kappa)$ implies that $s-g-2 \leq \kappa$. Since $s \geq 2 g+1$, we get the inconsistent set of inequalities $g-1 \leq \kappa<g-1$. By Clifford's Theorem, we conclude that $\mathcal{O}_{H^{\nu}}(1)$ is non-special. By the Riemann-Roch Theorem, $h^{0}\left(\mathcal{O}_{H^{\nu}}(1)\right)=s-2 g+\kappa+1<s-g$. Since $H$ spans $\mathbb{P}^{s-g-1}$, this is a contradiction. We, therefore, conclude that $C$ cannot be a section of $X$.

Hence, by Lemma 2.1, we conclude that $C$ admits a two-to-one map to $B$, a curve of genus

$$
\frac{d+g-s+1}{2}=\frac{\kappa+1}{2} .
$$

Since the genus is an integer, we also conclude that $\kappa$ must be odd. This concludes the proof of Theorem 1.6.

We next make a few remarks about the locus of curves $C$ in the moduli space of curves $\mathcal{M}_{g}$ such that $C_{c a n}$ lies on a surface of degree $g+\kappa$. Recall the following well-known lemma.

Lemma 3.1. The locus of curves $C$ of genus $g \geq 2$ that admit a two-toone map to a curve of genus $h$ has codimension $g+h-2$ in the moduli space $\mathcal{M}_{g}$ of curves of genus $g$.

Proof. By the Riemann-Hurwitz Formula, a two-to-one map has $2 g-$ $4 h+2$ branch points. By the Riemann Existence Theorem, if we fix a curve $B$ of genus $h$ and $2 g-4 h+2$ branch points, then there exists a positive finite number of double covers $C_{i}$ of genus $g$ that are branched along the chosen points. Furthermore, a curve of genus $g \geq 2$ occurs only finitely 
many times in this way. We conclude that the dimension of the locus of curves of genus $g$ that admit a two-to-one cover of a curve of genus $h>1$ is $3 h-3+2 g-4 h+2=2 g-h-1$. Since the dimension of $\mathcal{M}_{g}$ is $3 g-3$, the codimension of this locus is $g+h-2$. When $h=0$ or 1 , this calculation has to be modified because curves of genus 0 and 1 have positive dimensional automorphism groups. A simple calculation shows that the dimension of the locus of hyperelliptic curves is $2 g-1$. Hence, the codimension is $g-2$. Similarly, the dimension of the locus of bi-elliptic curves is $2 g-2$. Hence, the codimension is $g-1$.

Corollary 3.2. Let $\kappa \geq-1$ be an odd integer. Let $g>\max (10,3 \kappa+$ 12). Then the codimension of the locus of curves $C$ in $\mathcal{M}_{g}$ such that the minimal degree surface in $\mathbb{P}^{g-1}$ containing $C_{\text {can }}$ has degree $g+\kappa$ is $g+\frac{\kappa-1}{2}$.

Proof. By Part (2) of Theorem 1.1, $C$ has to be a double cover of a curve of genus $\frac{\kappa+3}{2}$. By Part (3) of Theorem 1.1, every double cover of a curve of genus $\frac{\kappa+3}{2}$ is contained in a surface of degree $g+\kappa$. Hence, by Lemma 3.1, the codimension of the locus of curves in $\mathcal{M}_{g}$ such that the minimal degree surface containing $C_{c a n}$ has degree $g+\kappa$ is $g+\frac{\kappa+3}{2}-2=g+\frac{\kappa-1}{2}$.

\section{Low degree examples}

Smooth surfaces with small sectional genus have been classified by Ionescu [Io], Livorni $[\mathbf{L 1}],[\mathbf{L 2}]$ and in positive characteristic by Andreatta and Ballico $[\mathbf{A B}]$. In this section, using the classification of surfaces with small sectional genus, we give some examples of canonical curves of genus $g$ contained in surfaces of degree $g+\kappa$ when $g \leq 3 \kappa+12$.

We first specialize [CH, Lemma 1.3] to our case.

LEMmA 4.1. Let $-1 \leq \kappa \leq 5$. Let $S$ be the minimal degree surface with degree $g+\kappa<2 g-3$ containing a canonical curve $C_{\text {can }}$ of genus $g$. Then $S$ is birationally ruled.

Proof. First, suppose that $g+\kappa<2 g-4$. Then, by Clifford's Theorem, $\mathcal{O}_{H}(1)$ is non-special on a general hyperplane section $H$ of $S$. Let $h$ be the genus of $H$. Then, by the Riemann-Roch Theorem, $g+\kappa-h \geq g-2$. Therefore, by the genus formula,

$$
K_{S} \cdot H \leq 2 h-2-H^{2}=2 h-2-g-\kappa \leq \kappa-g-2 \leq-2 .
$$

Since $H$ is ample, no multiple of $K_{S}$ can have a section. By Enriques' Theorem $[\mathbf{B}], S$ is birationally ruled.

If $g+\kappa=2 g-4$, then $\kappa=g-4$. Hence, if $-1 \leq \kappa \leq 5$, then $3 \leq g \leq 9$, respectively. In Example 1.7, we saw that every canonical curve of genus $3,4,5,6$ or 7 is contained in a surface of degree less than or equal to $1,2,4,5,8$, respectively. A general curve of genus 8 can be realized as a $(5,5)$ curve on $\mathbb{P}^{1} \times \mathbb{P}^{1}$ with eight nodes. The linear system of $(3,3)$ curves vanishing on the nodes of $C$ maps the surface to $\mathbb{P}^{7}$ as a surface of degree 10 containing $C_{\text {can }}[\mathbf{A C G H}]$. Hence, every canonical curve of genus 8 is 
contained in a surface of degree less than or equal to 10. Similarly, a general curve $C$ of genus 9 can be realized as a plane degree eight curve with 12 nodes. The linear system of quintic curves vanishing at the nodes embeds the blow-up of $\mathbb{P}^{2}$ at the nodes as a surface of degree 13 in $\mathbb{P}^{8}$ containing $C_{c a n}$. Hence, every canonical curve of genus 9 is contained in a surface of degree at most 13. Therefore, in all these cases the minimal degree surface containing $C_{c a n}$ has degree strictly less than $2 g-4$. This concludes the proof of the Lemma.

REMARK 4.2. When $\kappa \geq 6$, in addition to birationally ruled surfaces, we would need to allow K3 surfaces $[\mathbf{C H}]$.

For the rest of this section, let $-1 \leq \kappa \leq 3$ and $g \leq 3 \kappa+12$. Suppose that the minimal degree surface $S$ containing $C_{\text {can }}$ has degree $g+\kappa<2 g-3$. For simplicity, we will assume that $S$ is smooth. The smoothness assumption is for convenience and can be removed. If $S$ is a singular surface of degree $d$ in $\mathbb{P}^{n}$, then the projection of $S$ from a singular point is either a curve, in which case $S$ is a cone, or is a surface of degree less than or equal to $d-2$ in $\mathbb{P}^{n-1}$. Successively projecting $S$ from singular points leads to an analysis of the singular case as well. We leave this analysis to the interested reader.

Let $F_{r}, r \geq 0$, denote the ruled surface $\mathbb{P}\left(\mathcal{O}_{\mathbb{P}^{1}} \oplus \mathcal{O}_{\mathbb{P}^{1}}(r)\right)$ over $\mathbb{P}^{1}$. Let $E$ denote the class of the curve with minimal self-intersection. Let $F$ denote the class of a fiber of the projective bundle.

- $\kappa=0$. Suppose that the minimal degree surface $S$ containing $C_{c a n}$ is smooth and has degree $g$. By Clifford's Theorem, the hyperplane section of $S$ can have genus at most 2 . In fact, by del Pezzo's classification $[\mathbf{L} 1$, Theorem 0.2], the hyperplane section must have genus 2. By the classification of surfaces whose hyperplane sections have genus $2[\mathbf{L} \mathbf{1}$, Table $]$ and $[\mathbf{A B}$, Table], we conclude that if $S$ is not a scroll, then $S$ must be a blow-up of $F_{r}$ with $0 \leq r \leq 2$ in $m \leq 7$ points embedded in $\mathbb{P}^{11-m}$ by the linear system $\left|2 E+(r+3) F-\sum_{i=1}^{m} E_{i}\right|$, where $E_{i}$ denote the classes of the exceptional divisors. If we take a curve $C$ on $S$ with class $4 E+(2 r+5) F-\sum_{i=1}^{m} 2 E_{i}$, then $C$ is a curve of genus $12-m$ that embeds in $\mathbb{P}^{11-m}$ as a canonical curve. In particular, note that the projection from $S$ to $\mathbb{P}^{1}$ defines a $g_{1}^{4}$ on $C$. This classification agrees with the one given in $[\mathbf{C a}]$.

- $\kappa=1$. Suppose that the minimal degree surface $S$ containing $C_{c a n}$ is smooth and has degree $g+1$. Then, by Clifford's Theorem, a hyperplane section of $S$ can have genus at most 3. Since in Example 1.7 we have analyzed canonical curves of genus $g \leq 7$, we may assume that $15 \geq g \geq 8$. If the hyperplane section of $S$ has genus 2 , then by the classification of surfaces whose hyperplane sections have genus 2 [L1, Table] and [AB, Table], $S$ has to be a scroll. In the proof of Theorem 1.1, we have seen that $C$ has to be a double cover of a curve $B$ of genus 2 and $S$ is the image of the surface $X=\mathbb{P}\left(f_{*} K_{C}\right)$ under the linear system $\left|\mathcal{O}_{X}(1)\right|$. In fact, since $B$ is hyperelliptic, $S$ is not smooth in this case. 
We may assume that hyperplane sections of $S$ have genus 3 and that $S$ is not a scroll. By the classification of surfaces whose hyperplane sections have genus 3 [L1, Table] and [AB, Table], there are two possibilities. First, $S$ may be the blow-up of $\mathbb{P}^{2}$ in $15-g$ points embedded in $\mathbb{P}^{g-1}$ by the linear system $\left|4 H-\sum_{i=1}^{15-g} E_{i}\right|$, where $H$ denotes the pull-back of the hyperplane class of $\mathbb{P}^{2}$ and $E_{i}$ denote the classes of the exceptional divisors of the blowup. In this case $C$ has class $7 H-2 \sum_{i=1}^{15-g} E_{i}$ and embeds in $\mathbb{P}^{g-1}$ as a canonical curve of genus $g$.

Second, $S$ may be a blow-up of $F_{r}$ with $0 \leq r \leq 3$ in $15-g$ points embedded in $\mathbb{P}^{g-1}$ by the linear system $\left|2 E+(r+4) F-\sum_{i=1}^{15-g} E_{i}\right|$. Curves in the class $4 E+(2 r+6) F-2 \sum_{i=1}^{15-g} E_{i}$ are mapped to canonical curves of genus $g$ under this linear system. Note that both types of curves are very special in moduli. For example, curves of the first type admit a $g_{7}^{2}$ and curves of the second type admit a $g_{4}^{1}$.

REMARK 4.3. After I wrote this paper, I became aware that Casnati in $[\mathbf{C a 2}]$, independently and using different techniques, classified canonical curves of genus $g$ that are contained in surfaces of degree $g+1$. Unlike here, Casnati does a very careful analysis of the singular surfaces as well. The classification remains essentially the same. Assuming that $g \leq 15$ and the surface is not a scroll, the curve is either birational to a plane septic with $15-g$ possibly infinitely near double points as in our first case or it lies in a conic bundle over $\mathbb{P}^{1}$ and hence admits a $g_{4}^{1}$ as in our second case (see Theorem D $[\mathbf{C a 2}])$.

- $\kappa=2$. Suppose that the minimal degree surface $S$ containing $C_{c a n}$ is smooth and has degree $g+2$. A general curve of genus 8 can be realized as a $(5,5)$ curve on $\mathbb{P}^{1} \times \mathbb{P}^{1}$ with eight nodes. The linear system of $(3,3)$ curves vanishing on the nodes of $C$ maps the surface to $\mathbb{P}^{7}$ as a surface of degree 10 containing $C_{c a n}[\mathbf{A C G H}]$. Hence, every canonical curve of genus 8 is contained in a surface of degree at most 10 . We may, therefore, assume that $18 \geq g>8$. By Clifford's Theorem, a hyperplane section of $S$ can have genus at most 4 . Since in this case the surface cannot be a scroll, by the classification of surfaces whose hyperplane sections have genus at most $4[\mathbf{L} \mathbf{1}$, Table $]$ and $[\mathbf{A B}$, Table], we conclude that the hyperplane section of $S$ must have genus 4 . The following can be deduced from [L1, Table] and [AB, Table].

First, $S$ can be the blow-up of $F_{r}$, with $0 \leq r \leq 4$, in $18-g$ points embedded in $\mathbb{P}^{g-1}$ by the linear system $\left|2 E+(r+5) F-\sum_{i=1}^{18-g} E_{i}\right|$. Curves in the class $4 E+(2 r+7) F-2 \sum_{i=1}^{18-g} E_{i}$ are mapped to canonical curves of genus $g$ under this linear system. If $g>16$, then this is the only other possibility.

Second, $S$ can be the blow-up of $\mathbb{P}^{1} \times \mathbb{P}^{1}$ in $16-g$ points embedded in $\mathbb{P}^{g-1}$ by the linear system $\left|\mathcal{O}_{\mathbb{P}^{1} \times \mathbb{P}^{1}}(3,3)-\sum_{i=1}^{16-g} E_{i}\right|$. Curves in the class 
$\mathcal{O}_{\mathbb{P}^{1} \times \mathbb{P}^{1}}(5,5)-2 \sum_{i=1}^{16-g} E_{i}$ map to canonical curves of genus $g$. This is the only other possibility if $g>10$.

Third, if $g=10$ (respectively, 9), $S$ may be the two-uple Veronese embedding of a cubic surface (respectively, of the blow-up of a cubic surface in a point $p$ ) in $\mathbb{P}^{3}$. Under this embedding complete intersections of two cubic surfaces (respectively, those that are double at $p$ ) in $\mathbb{P}^{3}$ map to canonical curves of genus 10 (respectively, 9).

Note that each of these curves are very special in moduli. The curves of the first type admit a $g_{4}^{1}$. The curves of the second type admit a $g_{5}^{1}$. Finally, curves of the third type are complete intersections of two cubic surfaces in $\mathbb{P}^{3}$.

- $\kappa=3$. Suppose the minimal degree surface $S$ containing $C_{c a n}$ is smooth and has degree $g+3$. For simplicity, we will assume that $21 \geq g>9$. By Clifford's Theorem a hyperplane section of $S$ has genus at most 5 . If the genus is 3, then $S$ is a scroll over a curve $B$ of genus 3 and $C$ has to be a double cover of $B$. This case has been studied in the proof of Theorem 1.1, so we may assume that $S$ is not a scroll. Then, by the classification in $[\mathbf{L 1}]$ and $[\mathbf{A B}]$, we conclude that a hyperplane section of $S$ has genus 5 . The possibilities can be read off from these tables.

First, $S$ may be the blow-up of $F_{r}$ with $0 \leq r \leq 5$ in $21-g$ points, embedded in $\mathbb{P}^{g-1}$ by the linear system $\left|2 E+(r+6) F-\sum_{i=1}^{21-g} E_{i}\right|$. Curves in the class $4 E+(2 r+8) F-2 \sum_{i=1}^{21-g} E_{i}$ are mapped to canonical curves of genus $g$ under this linear system. If $g>18$, then this is the only possibility.

Second, $S$ may be the blow-up of $F_{1}$ in $18-g$ points, embedded in $\mathbb{P}^{g-1}$ under the linear system $\left|3 E+5 F-\sum_{i=1}^{18-g} E_{i}\right|$. Curves in the class $\mid 5 E+$ $8 F-2 \sum_{i=1}^{18-g} E_{i} \mid$ map to canonical curves of genus $g$ under this embedding. This is the only other possibility if $g>13$.

Let $D$ be the del Pezzo surface obtained by blowing up $\mathbb{P}^{2}$ in 5 points. Denote the classes of the exceptional divisors by $A_{i}$. Third, $S$ may be the blow-up of $D_{5}$ in $13-g$ points embedded in $\mathbb{P}^{g-1}$ by the linear system $\left|6 H-2 \sum_{i=1}^{5} A_{i}-\sum_{i=1}^{13-g} E_{i}\right|$. Curves in the class $9 H-3 \sum_{i=1}^{5} A_{i}-2 \sum_{i=1}^{13-g} E_{i}$ map to canonical curves of genus $g$. These are the only possibilities for $g>9$.

Observe that these curves are very special. In the first case, the curves admit a $g_{4}^{1}$. In the second case, the curves admit a $g_{5}^{1}$. In the final case, the curve admits a $g_{9}^{2}$ with 5 triple points.

REMARK 4.4. In these examples, the assumption that $S$ is smooth rules out certain singular conic bundles over $\mathbb{P}^{1}$ or an elliptic curve that contain canonical curves. Most importantly, when $\kappa=2$ and $g \leq 10$ or $\kappa=3$ and $g \leq 13$, there are canonical curves that are triple covers of elliptic curves that lie on singular conic bundles over the elliptic curve. See Remark 4.7 for more details on these surfaces.

This concludes the classification of canonical curves of genus $g$ that are contained in a smooth surface of degree $g+\kappa$ for $-2 \leq \kappa \leq 3$. This 
classification can be carried out for several more values of $\kappa$. However, the list of possibilities grows rapidly and quickly becomes unwieldy. Since these examples already illustrate the technique, we conclude the discussion here. The reader should observe that, just like in Theorem 1.1, if we assume that the genus is relatively large compared to $\kappa$, then the number of possibilities is small. The following proposition makes the next case after Theorem 1.1 more precise.

Proposition 4.5. Let $0<\kappa<g-3$ be an integer. Assume that

$$
3 \kappa+12 \geq g>\max (2 \kappa+12,3 \kappa+4) \text { and }(g, \kappa) \neq(15,2),(21,4) .
$$

If the minimal degree surface $S$ containing a canonical curve $C_{\text {can }}$ of genus $g$ has degree $g+\kappa$ and $S$ is not projectively ruled, then $S$ is a conic bundle over $\mathbb{P}^{1}$ and $C$ admits a $g_{4}^{1}$.

Proof. Let $X$ be the minimal desingularization of $S$ and let $L$ be the pull-back of $\mathcal{O}_{\mathbb{P}^{g-1}}(1)$ on $X$. We compute the $\Delta$-invariant of $X$ as follows

$$
\Delta=\Delta(X, L)=g+\kappa+2-g=\kappa+2 .
$$

Hence, by assumption

$$
\frac{1}{2}\left(h^{0}(X, L)-8\right) \geq \frac{1}{2}(g-8)>\kappa+2=\Delta .
$$

By $[\mathbf{H o}$, Theorem B], $S$ is either projectively ruled or ruled by conics. The statement of Theorem B in $[\mathbf{H o}]$ forgets to omit the two exceptions $(X, L)=\left(\mathbb{P}^{2}, \mathcal{O}_{\mathbb{P}^{2}}(4)\right)$ and $\left(\mathbb{P}^{2}, \mathcal{O}_{\mathbb{P}^{2}}(5)\right)$. Plane curves of degree 7 (respectively, 8) are mapped to canonical curves under the linear system $\left|\mathcal{O}_{\mathbb{P}^{2}}(4)\right|$ (respectively, $\left|\mathcal{O}_{\mathbb{P}^{2}}(5)\right|$ ). These canonical curves are contained in the fouruple, respectively, five-uple Veronese embedding of $\mathbb{P}^{2}$. This explains the need to exclude the cases $(g, \kappa)=(15,2)$ and $(21,4)$ in the statement of the proposition.

Since by assumption, $S$ is not projectively ruled, we conclude that $S$ is a conic bundle over a curve. In order to reach this conclusion, we did not need to assume that $g>3 \kappa+4$. By [Ho, Corollary $1.8(2)]$, we have that

$$
g \leq h^{0}(X, L) \leq 3 \Delta+6-8 h^{1}\left(X, \mathcal{O}_{X}\right)=3 \kappa+12-8 h^{1}\left(X, \mathcal{O}_{X}\right) .
$$

If $g>3 \kappa+4$, then $h^{1}\left(X, \mathcal{O}_{X}\right)=0$ and $X$ and $S$ are rational surfaces. In particular, $S$ is a conic bundle over $\mathbb{P}^{1}$ and the projection of $C_{c a n}$ to $\mathbb{P}^{1}$ gives a $g_{4}^{1}$ on $C$. Observe that since the degree of the minimal surface containing $C_{c a n}$ is greater than $g-2, C$ cannot be trigonal. This concludes the proof of the proposition.

REMARK 4.6. More generally, by the same argument, if $3 \kappa+12 \geq g>$ $\max (2 \kappa+12,3 \kappa+4-8 i)$ and the minimal degree surface $S$ containing $C_{c a n}$ has degree $g+\kappa$ and is not projectively ruled, then $S$ is a conic bundle over a curve $B$ of genus at most $i$. The projection of $S$ to $B$ defines a map of degree at most 4 from $C$ to $B$. 
REMARK 4.7. The bounds in Proposition 4.5 are sharp. Assume that $\kappa \geq 2$ is even. Let $C$ be a curve in the linear system $\left|\mathcal{O}_{\mathbb{P}^{1} \times \mathbb{P}^{1}}\left(5, \frac{\kappa}{2}+4\right)\right|$. Then $C$ is a curve of genus $2 \kappa+12$. The linear system $\left|\mathcal{O}_{\mathbb{P}^{1} \times \mathbb{P}^{1}}\left(3, \frac{\kappa}{2}+2\right)\right|$ embeds $\mathbb{P}^{1} \times \mathbb{P}^{1}$ as a surface $S$ of degree $3 \kappa+12$ and restricts to the canonical embedding on $C$. In particular, $S$ does not contain any conics and a general curve $C$ in the linear system does not admit a $g_{4}^{1}$. To get examples when $\kappa$ is odd, one can repeat the construction with the surface $F_{1}$ instead of $\mathbb{P}^{1} \times \mathbb{P}^{1}$.

Let $S^{\prime}$ be a cone over an elliptic normal curve of degree $r$ in $\mathbb{P}^{r}$. Let $C$ be the intersection of $S^{\prime}$ with a general cubic hypersurface. Then $C$ has genus $3 r+1$. The two-uple Veronese embedding of $S^{\prime}$ is a surface $S$ in $\mathbb{P}^{3 r}$ of degree $4 r$. This embedding restricts to the canonical embedding on $C$. Therefore, $\kappa=r-1$. In particular, $g=3 r+1=3 \kappa+4$. We conclude that $S$ is a conic bundle over an elliptic curve $B$ and $C$ is a triple cover of $B$.

\section{References}

[AB] M. Andreatta and E. Ballico. Classification of projective surfaces with small sectional genus: char $p>0$, Rend. Sem. Mat. Univ. Padova, 84 (1990), 175-193.

[ACGH] E. Arbarello, M. Cornalba, P.A. Griffiths and J. Harris. Geometry of Algebraic curves, vol. 1, Grundlehren der mathematischen Wissenschaften 267, Springer-Verlag, 1984.

[B] A. Beauville. Complex algebraic surfaces. London Math. Soc. Student Texts 34. Cambridge University Press, 1996.

[Be] E. Bertini. Introduzione alla geometria proiettiva degli iperspazi Enrico Spoerri, Pisa, 1907.

[Ca] G. Casnati. Canonical curves on surfaces of very low degree. Proc. Amer. Math. Soc. 140 (2012), 1185-1197.

[Ca2] G. Casnati. Curves of genus $g$ whose canonical model lies on a surface of degree $g+1$. to appear Proc. Amer. Math. Soc.

[CH] C. Ciliberto and J. Harris. Surfaces of low degree containing a general canonical curve. Comm. Algebra 27 (1999), 1127-1140.

[D] Del Pezzo. Sulle superficie di ordine $n$ immerse nello spazio di $n+1$ dimensioni, Rend. Circ. Mat. Palermo, 1, 1886.

[EH] D. Eisenbud, and J. Harris. On varieties of minimal degree (a centennial account) Algebraic geometry, Bowdoin 1985 Proc. Sympos. Pure Math. 46 no. 1, Amer. Math. Soc., Providence, RI, 1987.

[F1] T. Fujita. Classification of projective varieties of $\Delta$-genus one. Proc. Japan Acad. Ser. A 58 (1982), 113-116.

[F2] T. Fujita. On polarized manifolds of $\Delta$-genus two. J. Math. Soc. Japan 36 (1984), 709-730.

[F3] T. Fujita. Classification theories of polarized varieties, London Math. Soc. Lecture Notes Series 155, Cambridge University Press, 1990.

[F4] T. Fujita. On the structure of polarized varieties with $\Delta$-genus zero, J. Fac. Sci. Univ. of Tokyo, 22 (1975), 103-115.

[H] R. Hartshorne. Algebraic geometry. Springer, 1977.

[Ho] T. Horowitz. Varieties of low $\Delta$-genus. Duke Math. J. 50 no. 3 (1983), 667-683.

[Io] P. Ionescu. Embedded projective varieties of small invariants. Algebraic geometry, Bucharest 1982. Lecture Notes in Math. 1056, Springer, Berlin, 1984.

[L1] E.L. Livorni, Classification of algebraic surfaces with sectional genus less than or equal to six.I: Rational surfaces. Pac. J. Math. 113 no. 1 (1984), 93-114.

[L2] E.L. Livorni, Classification of algebraic non-ruled surfaces with sectional genus less than or equal to six. Nagoya Math. J. 100 (1985), 1-9.

University of Illinois at Chicago, Department of Mathematics, Statistics and ComPuter Science, Chicago, IL 60607

E-mail address: coskun@math.uic.edu 